\title{
On Dealing with the Innovations of the Future
}

\author{
Raymond E. Spier ${ }^{1}$
}

Received: 9 March 2015/Accepted: 10 March 2015/Published online: 20 March 2015

(C) Springer Science+Business Media Dordrecht 2015

They may not have happened yet; but they are on the way. Reports, conference talks and exhibitions have provided windows into our possible and probable futures. As our ways of living have changed dramatically over the last 20 or so years, so might we expect even more such changes in the next couple of decades? But what changes might be in the offing and how should we as citizens, students, educators, ethicists and concerned individuals deal with them?

Not all of the prospects that are in today's news will emerge and become consolidated as way-of-life changing mediators. Some of the developments that are on the cards may be difficult to scale-up, produce or manufacture. Others will fall by the wayside because of cost issues, lack of financial backing or poor marketing. And there are always issues of safety, real or imagined, to be dealt with as well as the ethical issues entailed. Also it must be appreciated that there is a category difference between the production of a "tool" and the "tasks" to which that tool is applied. We know full well that a hammer is an acceptable tool when knocking in nails for the construction of a useful artefact but that it can also be used for taking human life.

Notwithstanding the hurdles that have to be jumped, we can be sure that new developments in ever larger numbers will succeed in running the gauntlet of criticism, rejection and fear of the new. Some will emerge without severe scrutiny by society at large while others will struggle for years before becoming accepted. The amount of change that will be engendered by the emergent inventions and developments will not be contingent on the level of difficulty they have in becoming part of our everyday lives.

Raymond E. Spier

r.spier@surrey.ac.uk

1 University of Surrey, Guildford, UK 
It is possible to illustrate this by considering the ways humans have gone about the production and use of adding machines. There is little doubt that the use of fingers, toes, hands and feet have been of material assistance in the process of helping humans to add quantities of things together. The abacus came into use in Babylon some 4500 years ago and is still used in the Far East. The nineteenth century hosted the emergence of the first machines that could compute; initially it was the Englishman, Charles Babbage (1791-1871) and his son, helped on the programming side by Augusta Ada Lovelace (1815-1852) who designed, built and ran mechanical machines that could do calculations based on a program of instructions. But it was Alan Turing (1912-1954) who instigated in 1936 the notion that any number or letter could be expressed as a string of zeros and ones and that such strings could be manipulated to do calculations that were set out in the form of a program. In the short period of 12 years developments of these concepts led, at the University of Manchester (UK), to the 1948 demonstration by Frederic C. Williams, Tom Kilburn and Geoff Tootill of a universal computer that used a stored program. Such a computer was capable of some 5000 calculations a second. Developments in electronics since that time have led to a machine (located in Guangzhou, China) that, in 2015, can do some 33,800 trillion mathematical operations per second (33.8 Petaflops). These speeds are set to double every 2 years.

So, it is not surprising that the "MIT Technology Review" (March/April, 2015) when highlighting "Ten Breakthrough Technologies" selected four topics involving computers. These include the installation of within-car computing and communication capabilities that enable vehicles to communicate their positions, directions and speeds to one another against a road network background. It is expected that such devices will, in the near-term, decrease the number of vehicular collisions. A second application is the introduction of the "Apple Pay", a device that enables secure payments for goods and services. Thirdly, computers have been put to the task of creating the illusion of a 3D-reality, a virtual-reality, which gives the viewer some control over the appearance of the images of this computer-created state of being. And fourthly, people are working on the storage of all the data derived from the sequencing of the DNA of human genomes in a "cloud" based situation such that this mass of sequences can become available to all those who wish to compare human genomes to better diagnose disease and nominate cures. This process requires the comparison of hundreds of petabytes $\left(10^{15}\right)$ of data per year.

Once this computing power is packed into a robot, a whole new world emerges. The journal "Science" provided a special section on this topic in its 10th October 2014 Issue (\#346). Others have also been addressing the prospects of robots having intellectual capabilities that exceed those of humans. (We have already seen computers beating world champion chess players, or winners of quiz games.) And the ability of one's hand-held smart phone to translate speech and handwriting into machine text is a marvel of programming. As these electronic capabilities are increasing daily, the use of machines to do all the tasks that humans presently perform is close to being realized. How humans in their querulous societies create regulatory environments (ethics) that harness these robotic powers for the benefit of humans will become one of the greatest challenges that our species will face. 
While digital developments proceed apace there are parallel developments in the world of materials. The ability to make substances whose structures, compositions and properties are controlled at the nano-level $\left(10^{-9}\right.$ of a meter $)$ is showing signs of leading to stronger and more flexible materials that can be used to crack one of the major problems in the storage of electricity - the all-purpose, cheap and hardy battery, capable of higher energy densities than those presently available ("MIT Technology Review", Mar/Apr 2015). Indeed, a recent issue of "New Scientist" (11th Oct, 2014, issue 2990) highlights the use of glass to store information more securely than current integrated circuit technology; a combination of shrimp shells and spiders web silk can be used as a biodegradable plastic that can substitute for present synthetic materials; there are possible low energy microcircuits made of Stanene that will use less energy than silicone based chips; new aerogels can be used as cleaners, and materials can be created that can self-regenerate when chipped or scratched; other new substances called Skutterudites enable the recycling of the energy released from working machines, while woods can be made so strong that skyscrapers may be built from them. As these developments are coming to the fore there is the emergence of new compositions for medically applied prostheses and the devices that enable more reliable surgery and post-operational care: tubes, bags, pumps, sutures and physiologically active implements.

Growing human cells in vitro is becoming an everyday practice. There are many ways of producing such cells including the practice of inducing stem cell capability from otherwise normal somatic cells. These are early days for the application of stem cells to cure diseased individuals but reports of successful therapies are increasing in number daily. Cell culture techniques have now advanced to the stage when it is possible to grow clumps of nerve cells that show rudimentary differentiation into crude organ preparations with elements of brain-like structures ("MIT Technology Review", Mar/Apr 2015). Such entities when further developed may serve as brain analogues that may be used to discover how brains function. May this not lead to ethical questions concerning the creation of a conscious entity in a petri dish? This could lead to the addition of a biological brain to a mechanical entity (See "Davros" in the "Dr. Who" television serial in UK 1963-1989 and 2005-present-813 episodes).

Additional advances in human medicine may derive from the more sophisticated testing of human blood samples and other liquid exudates from the human body. The automation of such tests and their adaptation to bedside and immediate test devices are also goals that are being pursued with all due speed. These diagnostic developments coupled with DNA sequences are the foothills of radical changes heralding a new era of medicine based on molecular knowledge with accompanying therapeutic interventions.

Extending the fields where new and game-changing developments may be expected, readers can note Google's development of balloons ("Loons") to carry the internet to parts of the globe that cannot make contact via the Web to the rest of the world. Necessary advances in methods of desalination of sea or brackish water are proceeding rapidly, particularly in arid regions. This facility may be used in conjunction with another major advance in being able to genetically engineer food 
crop plant species to more efficiently absorb carbon dioxide from the atmosphere, thereby increasing food yields by $50 \%$.

These advances are expected with a high degree of confidence and there are many more such advances that are already in train. Also, for all the advances that have made themselves known, there are an equivalent number or more that are below the threshold of public awareness.

To take full advantage of this pipe-line of upcoming opportunities which project human societies into the big unknown there will have to be changes to the way in which societies deal with these new challenges. The misapplication of the "Precautionary Principle" can be deployed to delay, deny and denigrate the opportunities for advancement and progress. This should be avoided. Yes, the adoption of the new has to have a regard to both the safety and value aspects of that novelty. It is owed to those yet unborn that as the current occupiers of the human biosphere venture into new areas, they have to be courageous, daring and resolute in accepting the challenges of the changing world ahead. 